\title{
Heterogeneous cellular and DSRC networking for Floating Car Data collection in urban areas
}

\author{
Pierpaolo Salvo $^{\mathrm{a}}$, Ion Turcanu ${ }^{\mathrm{a}}$, Francesca $\mathrm{Cuomo}^{\mathrm{a}}$, Andrea Baiocchi ${ }^{\mathrm{a}, *}$, Izhak Rubin ${ }^{\mathrm{b}}$ \\ ${ }^{a}$ Dept. of Information Engineering, Electronics and Telecommunications \\ Sapienza University of Rome, Via Eudossiana 18, 00184, Rome, Italy \\ ${ }^{b}$ Electrical Engineering Department, UCLA, Los Angeles, CA, USA
}

\begin{abstract}
Vehicular traffic monitoring is a major enabler for a whole range of Intelligent Transportation System services. Real time, high spatial and temporal resolution vehicular traffic monitoring is becoming a reality thanks to the variety of communication platforms that are being deployed. Dedicated Short Range Communications (DSRC) and cellular communications like Long Term Evolution (LTE) are the major technologies. The former is specifically tailored for Vehicular Ad-hoc Network, the second one is pervasive. We propose a fully distributed Floating Car Data (FCD) collection protocol that exploits the heterogeneous network provided by DSRC and LTE. The proposed approach adapts automatically to the penetration degree of DSRC, achieving the maximum possible LTE offloading, given the VANET connectivity achieved via DSRC. Extensive simulations in real urban scenarios are used to evaluate the protocol performance and LTE offloading, as compared to baseline and literature approaches.
\end{abstract}

Keywords: Intelligent Transportation Systems, Vehicle-to-vehicle/roadside/Internet communication, Applications

\section{Introduction}

Floating Car Data (FCD) are an essential input to an increasing number of applications in the context of the Intelligent Transportation System (ITS) [1][2]. Under the ETSI definition of Cooperative Awareness Basic Service [3], Cooperative Awareness Messages (CAMs) are exchanged in VANETs to promote and maintain awareness among vehicles and to support cooperative interactions among networked vehicles. Such messages provide positional information, as well as identify the status of neighboring vehicles. Each vehicle records and updates its Local Dynamic Map (LDM), where information collected about neighboring vehicles are stored. Due to vehicles' mobility, this information is updated periodically through the exchange of CAMs.

Despite the recommendation made in 2011 of using the IEEE 802.11p [4] protocol as the standard for vehicular communications, in recent years many researchers and industrial organizations have considered using the LTE cellular network as an alternative solution for vehicular networking applications, specifically for the transport of FCD message flows.

While cellular communications and specifically LTE is a viable solution for collating data sensed by roaming vehicles, there is an obvious interest in exploiting the $75 \mathrm{MHz}$

\footnotetext{
*Corresponding author

Email address: andrea.baiocchi@uniroma1.it (Andrea Baiocchi)
}

bandwidth allocated to vehicle-to-everything (V2X) communications, by profiting of the On Board Units that are expected to be installed on board vehicles, pushed by safety considerations (e.g., see [5]).

In this paper, considering an urban scenario, we present a hybrid networking mechanism under which a VANET based vehicle-to-vehicle (V2V) networking protocol is employed for the purpose of supporting LTE based FCD collection operation. A preliminary work on our proposal has been presented in [6]. The aim is to substantially reduce the number of concurrently active LTE channels and the information message load carried across the LTE cellular network for the same accuracy of the vehicular traffic description obtainable when FCD are collected via LTE from each individual vehicle. We define a distributed procedure that exploits the "horizontal" capability of vehicles to communicate among themselves via the VANET, to elect representative nodes. The election process exploits the logic of the so called dissemination protocols. The representative nodes are responsible for communicating aggregated FCD via the LTE infrastructure. The performance gains achieved through the use of the proposed approach rapidly increase as the vehicular density increases. Under such high density conditions, the traffic load of the LTE cellular network can become critically high, while VANET networking connectivity improves. Under low vehicular density levels, our procedure falls back onto the use of a plain LTE-based FCD collection scheme. The employed operation and protocols rely on the use of geographical information known individually by each vehicle (e.g., via 
GPS), not requiring the use of external databases (such as those that make use of urban city maps and junction proximity sensors).

The rest of the paper is structured as follows. The innovation and complementarity of our approach with respect to other hybrid VANET and LTE procedures, that have been published to date, are discussed in Sec. 2. The details of the proposed protocol are defined in Sec. 3. The simulation model used in the performance evaluation of the proposed approach is described in Sec. 4. In Sec. 5, we present illustrative performance results. Conclusions are drawn in Sec. 6.

\section{Hybrid VANET-LTE floating car data collection scenario}

We consider an urban area scenario covered by one or more LTE macro-cells. FCD updates originated by vehicles moving in the underlying coverage area are collected continuously over time and fed to a number of ITS related applications. Conceptually, we think of the collected FCD as processed by a backhaul server, referred here to as $F C D$ processing server (FPS). The placement of the FPS (in a remote data center or close to the monitored area) is immaterial to the ensuing discussion. The relevant point is that FCD collected from the monitored area, often encompassing more than a single LTE macro-cell, are processed together, thus exploiting jointly the information collected over the entire monitored area.

Vehicles are assumed to be equipped with On Board Units (OBUs) supporting LTE, IEEE 802.11p, plus a GPS device. Vehicles generate, send and receive Cooperative Awareness Messages (CAMs) periodically, as described by the ETSI standard. The CAM exchange is conducted through the 802.11p VANET operation over its dedicated bandwidth [4]. By receiving CAMs, each vehicle creates its own Local Dynamic Map (LDM). In this manner, it is aware of the states of other vehicles in its neighborhood area, including their time-stamped positions, velocities, moving directions, vehicle attributes.

In the following we review the related literature (Sec. 2.1), then we present a general framework that encompasses most of the solutions proposed for FCD collection via VANET plus cellular network (Sec. 2.2). Finally, we outline our contribution (Sec. 2.3).

\subsection{Related works}

LTE-centric transport mechanism have been investigated, where FCD are collected from vehicles directly, by using on-board LTE radio modules. FCD can be collected directly with LTE, by requiring that each vehicle sends its own data periodically via an individual LTE channel. The same LTE network is then also used to disseminate this information in an area of interest. Calabuig et al. [7] give a detailed evaluation of LTE uplink and downlink traffic load generated by specific ITS applications, including FCD collection for vehicular traffic monitoring. The resulting load could become massive [8][9], so that even ad hoc planning of the LTE Radio Access Network could be required [8]. Hence, it makes full sense to exploit the bandwidth resource assigned to V2V Dedicated Short Range Communications (DSRC) alleviate the load on the cellular network.

Araniti ed al. [10] and Hammed et al. [11] compare VANET and LTE technologies, identifying the strengths and weaknesses of these two approaches under different conditions (vehicular density, vehicular speed, transmission rate). Candidate wireless technologies are reviewed and critical issues for LTE to support ITS application requirements are discussed in [10]. Extensive simulations presented in [11] make the authors conclude that the IEEE 802.11p offers acceptable performance for network topologies with limited mobility support. On the other hand, LTE meets most of the application requirements in terms of reliability, scalability, and mobility support; on the contrary, delay requirements appear to be critical, especially under high cellular network traffic load.

On the opposite side, VANET based traffic data collection has also been investigated (e.g., see [12][13]). A traffic monitoring system, named ABEONA, is proposed and experimented by Gramaglia et al. [12]. ABEONA achieves a distributed knowledge of vehicular traffic conditions and it is able to forecast short-term traffic over $15-20 \mathrm{~min}$ time windows, based on the assumption of VANET equipment availability. A data collection protocol named COL is defined by Dieudonne et al. [13]. COL works on demand and stops when the data collection is achieved. Experiments are presented to assess its capability to overcome network topology changes. COL assumes that vehicles are equipped with VANET technology.

An intermediate approach is represented by the employment of a heterogeneous network paradigm, identified also as a Hybrid Wireless Network [14] [15] [16] [17] [18]. This approach integrates the use of LTE cellular wireless communications technology with the IEEE 802.11p based VANET. The LTE4V2X system presented by Remy et al. [14] uses LTE technology to create clusters of vehicles. The latter are subsequently managed by using an IEEE 802.11p based VANET networking operation. A similar approach is adopted by Jia et al. [15], who study the impact of the vehicular data collection in an LTE network. There the cluster head selection process is managed by the base station node (eNodeB), making use of LTE communications channel quality indicators measured and reported by each vehicle. The authors show that such a system is able to reduce the negative impact of FCD load on the quality of the transport service obtained by conventional LTE traffic. Sivaraj et al. [16] propose a hybrid solution that entails the selection of the cluster head on the basis of different LTE parameters. D'Orey et al. [17] propose a centralized system for creating clusters and for electing cluster heads. The clustering process is performed here by a remote server, assuming it to have a much wider regional view of the system, when compared with the limited scope 


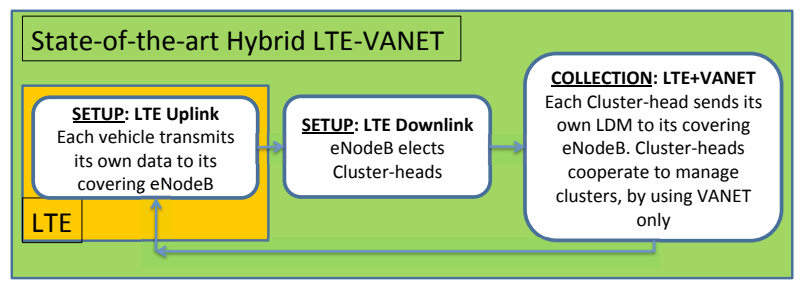

Figure 1: LTE and Hybrid LTE-VANET FCD collection schemes in the existing proposals.

available to a single eNodeB. Finally, De Felice et al. [18] propose a hybrid traffic monitoring service, named THOR, that combines VANET and LTE technologies in a standard compliant approach. Moreover, they propose an incident detection algorithm based on the information collected by THOR. However, their algorithm is designed only for highway scenarios, needing additional infrastructure elements (e.g., RSUs) to be operational. On the contrary, our proposed solution is designed for urban scenarios and does not require any additional infrastructure. Moreover, in this paper we generally deal with the use of hybrid VANETLTE for FCD, while the paper in [18] targets the use of LTE only for incident detection when the VANET is not available.

\subsection{State-of-the-art approach for FCD collection with a heterogeneous network}

All the state-of-the-art proposals reviewed above, based on a hybrid LTE plus VANET networking infrastructure, follow a common paradigm, where two main algorithmic phases can be recognized: i) SETUP; ii) COLLECTION (Fig. 1). The SETUP phase exploits the LTE cellular network, while the COLLECTION phase is based on both local communication in the VANET among neighboring vehicles and transmission of the collected data over LTE channels. If communication in the VANET is not possible, meaning that only the LTE technology is available, then SETUP and COLLECTION collapse in a unique phase. In this case FCD messages are collected individually by each vehicle (e.g., LTE box in Fig. 1).

The SETUP phase aims at gathering status information involving vehicles that roam in the target area, and making this information available to the FPS. Proposed techniques published to-date envision an operation during the SETUP phase under which each vehicle communicates the relevant data individually to the FPS, via LTE connections. This information is then used to set up, in an optimal fashion, the process governing the mode of operation to be used during the ensuing FCD COLLECTION phase.

During the COLLECTION phase, the vehicular population is split into clusters. Cluster head vehicles are elected based on the information collected during the SETUP phase. The choice of cluster head can be the outcome of an optimization problem that takes into account: i) information on vehicles' positions, velocities and directions; ii) VANET connectivity information (neighbors of each vehicle, according to the received CAMs); iii) information on the Channel Quality Indication (CQI) of LTE channels measured by the vehicle on board units. A centralised optimization approach, run in the FPS, can be used to identify the best candidate vehicles for the role of clusterhead nodes. The cluster heads are then designated at the end of SETUP phase, before the start of the ensuing COLLECTION phase, by sending control messages on the LTE downlink channels that cover the target area.

A cluster head is responsible for collecting FCD from its one-hop neighbouring vehicles via VANET wireless links. The cluster head then forwards the collected data to the FPS, using its LTE connection. In this manner, only cluster heads (rather then each vehicle) use LTE channels.

Let $N$ denote the number of vehicles ${ }^{1}$ in the target monitored area $A$. According to the above described operational paradigm, $N$ independent LTE channels are established and activated during the SETUP phase, while only $M \ll N$ LTE channels are used during the COLLECTION phase, where $M$ is the number of cluster heads. The average vehicular density is equal to $\rho=N / A$. If $R$ denotes the radio coverage range realized by a single nodal VANET transmitter, one can estimate the $M$ level to satisfy: $M \sim A /\left(\pi R^{2}\right)=N /\left(\rho \pi R^{2}\right)$. For a vehicle density of $\rho=100 \mathrm{veh} / \mathrm{km}^{2}$ and $R=300 \mathrm{~m}$, we have $M / N \approx 0.0354$.

In general $K \geq 1 \mathrm{FCD}$ collections are performed during a COLLECTION phase. The COLLECTION phase continues in an uninterrupted manner until it is determined that the current cluster layout deviates beyond a margin level from a currently calculated optimal configuration. A new SETUP phase is then triggered. The topological layout of cluster heads and their election operations are thus adapted to new system conditions, refreshing the information required to optimally synthesize the layout and operations governing the ensuing COLLECTION phase.

The duration of the COLLECTION phase is therefore tied to the scope and features of the monitored area and to the dynamics of the vehicular traffic roaming the area. Summing up, we envisage a time period $T_{\text {cycle }}$ to refresh the SETUP of the collection network. Within the time frame of duration $T_{\text {cycle }}$, one SETUP phase is carried out, with duration $T_{\mathrm{S}}$, as well as $K$ COLLECTION phases, each of duration $T_{\mathrm{C}}$. Then, it is $T_{\text {cycle }}=T_{\mathrm{S}}+K T_{\mathrm{C}}$.

To summarize there are different state-of-the-art approaches for both the SETUP and the COLLECTION. These can be summarized by a sort of flow chart (see Fig. 2 ). We can list the following different operations:

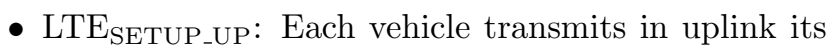
own data to its covering eNodeB;

\footnotetext{
${ }^{1} N$ is assumed to stay constant over one SETUP+COLLECTION
} cycle 


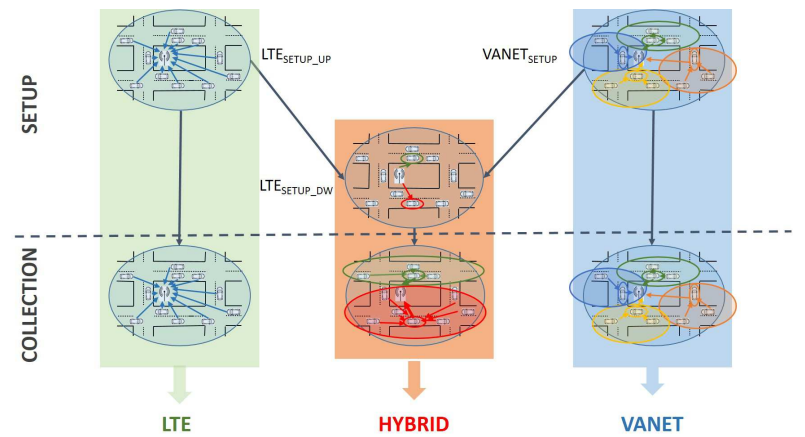

Figure 2: Summary of the three different schemes for SETUP and COLLECTION

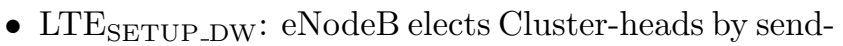
ing election messages in the downlink;

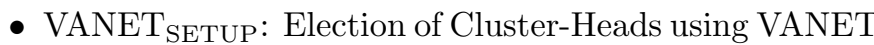
only; Each Cluster-head transmits its own partial LDM to its covering eNodeB;

- COLLECTION: Each node (in the LTE case) or Cluster-head (in the HYBRID or VANET cases) sends its own FCD and those of the vehicle nodes it is responsible for (if any) to its covering eNodeB.

\subsection{Our proposed approach (VANET $T_{S E T U P}$ and VANET in Figure 2)}

The lesson learned from the studies cited above is that the number of used LTE channels can be reduced by using only specifically designated nodes to send collected data through the LTE access network to the FPS. Each such designated node would aggregate and forward data that represents the status of vehicles in its immediate neighborhood. This status data stored in the LDM is available at each vehicle, as each one continuously collects such data through the maintenance of a background CAM exchange process.

The key idea of our proposal is that such designated nodes can be identified by executing an election process across the vehicular wireless network (VANET). The distributed protocol for the designated node election can be derived from the logic of dissemination protocols. A dissemination logic provides for the multi-hop transport of messages across the vehicular network through the election of certain vehicle nodes to act in forwarding a received message to other vehicles. By definition, the dissemination logic implies the designation of special nodes, that make up a connected set of nodes, covering the area spanned by the $V A N E T$. The designated nodes are employed as local data aggregation points that are used for collecting and sending FCD information obtained from neighboring vehicles to the FPS via LTE. The effectiveness of the dissemination procedure increases as the vehicular density increases. This is just the scenario where offloading for LTE access network is most critical.
A distinguishing feature of our proposal is that it is fully seamless for the LTE network. Differently from most previous approaches, no modification or new logic is required in the LTE cellular network. The designation of representative nodes and the local collection of FCD is carried out by a "horizontal" process that makes use of the VANET only. Elected representative nodes upload aggregated FCD to the FPS via LTE channels, without any further intervention from the LTE network, e.g., to orchestrate or manage vehicles clusters. This approach achieves a useful decoupling between vehicle specific functions (FCD aggregation and maintenance of up-to-date LDMs) and generic communication functions (uploading of FCD to the FPS via the LTE network). The proposed approach aims at leveraging the strong points of either technology: the VANET for its ease of direct communication among neighboring vehicles, the LTE access network for its potentially high capacity and pervasive availability. As a consequence the proposed approach adapts automatically to any given penetration rate of DSRC equipment.

Summing up, the key features of our proposed approach are as follows.

- We take advantage of utilizing the dedicated spectrum bands assigned for VANET services to reduce the traffic loads imposed on the LTE wireless access network.

- Our proposed mechanism can be realized in a manner that is fully compliant with current technology and standards, e.g., by using the CBF algorithm of the GeoNetworking protocol [19] as the dissemination logic. Alternatively, it could be programmed as an application level function sitting on top of standard PHY, MAC and network protocols.

- No special new function is required of the LTE cellular network, i.e., the proposed approach for FCD collection is fully seamless to the LTE network control plane.

- The LTE network, as well as other future cellular networks, can offer message transport at much higher communications rates. Cell sizes are becoming smaller and high inter-cell interference effects become dominant. The latter limits the attainable system throughput efficiency level. It is consequently more effective to employ a lower number of nodes for the forwarding of larger amounts of data aggregates, instead of a large number of sources of relatively small amounts of data.

\section{The VANET based protocol for the election of representative nodes}

\subsection{The dissemination logic}

Vehicle-to-vehicle multi-hop communications enable the extension of the road span covered by Road Side Units 
(RSUs) or On Board Units (OBUs) which act as data sources. Such a V2V multi-hop dissemination function is of interest for the rapid and effective transport of both safety and infotainment applications [20]. Geographical dissemination based techniques are surveyed in [21, Ch. $5][22]$.

The ETSI definitions of the GeoNetworking protocol [19] and network architecture [23] enable the multi-hop dissemination of messages in the VANET, merging the dissemination functionality into the vehicular networking layer and preserving the underlying $\mathrm{MAC}$ and $\mathrm{PHY}$ radio protocol layers as they are defined in the IEEE 802.11p. The Contention Based Forwarding (CBF) component of the GeoNetworking protocol (section E.3 of [19]) defines a timer-based dissemination logic for broadcast messages. A node $A$ receiving a message from node $B$, checks if it has already received and dealt with the received message. In case the message is new, $A$ sets a timer according to the value

$$
T= \begin{cases}T_{\max }-\left(T_{\max }-T_{\min }\right) \frac{d_{\mathrm{AB}}}{d_{\max }} & d_{\mathrm{AB}} \leq d_{\max } \\ \infty & d_{\mathrm{AB}}>d_{\max }\end{cases}
$$

where $T_{\max }$ and $T_{\min }$ are the maximum and minimum values set for the GeoNetworking broadcast message timer; $d_{\max }$ is the theoretical maximum communication range of the wireless access technology; $d_{\mathrm{AB}}$ is the distance between $A$ and $B$.

If $A$ receives more copies of the same message while the timer is running and $A$ 's copy of the message is scheduled for re-broadcasting, it cancels its scheduled copy and gives up to the re-broadcasting action (inhibition rule). If the timer in $A$ expires and $A$ does not get inhibited, then $A$ re-broadcasts the message. By doing so, $A$ is elected to be a forwarding node for that message. The dissemination algorithm suppresses most duplicate messages by electing designated nodes to act as forwarders.

Under our proposed mechanism, the forwarding nodes designated by the dissemination logic are used as a covering set of the vehicles, rather than to actually disseminate data flows. To underline the different purpose of the forwarding nodes in our scheme, we rename them as representative nodes. The representative nodes would then be used to send across the LTE network (to the FPS) status messages about themselves and about their neighbors (that they know about through the conduct of a background CAM exchange).

\subsection{Election of representative nodes: connected VANET case}

We define a REQUEST message that is originated by a trigger node, starting the dissemination-like process. The trigger node can be a RSU located in a central position of the target area, or it can be a designated OBU. The REQUEST message is disseminated according to the rules used by the GeoBroadcast protocol outlined in Sec. 3.1. The nodes that are elected as forwarders of the REQUEST message during this dissemination phase, are identified as representative nodes. They are in charge of reporting the status data of their neighboring vehicles to the FPS via LTE connections.

Let $A$ denote a generic node that sends the REQUEST message (hence $A$ is the trigger node or any of the elected representative nodes). The message sent by $A$ contains: i) an identifier; ii) the geographical position of $A$; iii) a count-down hop-count field, initialized by the trigger node to the maximum number of hops $H$ that the REQUEST message is allowed to travel and decremented by each rebroadcasting node; iv) a list $\mathcal{L}_{\mathrm{A}}$ of IDs of the vehicle nodes that $A$ commits to report to the FPS on.

By re-broadcasting the REQUEST message, a node $A$ recognizes to have been designated to act as a representative node for the nodes listed in $\mathcal{L}_{\mathrm{A}}$. Hence, the node $A$ constructs a reduced neighbor vehicle database $r L D M$ by omitting from its full LDM those nodes whose IDs are listed in the REQUEST message that $A$ has received. The list of IDs contained in the $r L D M$ is inserted in the copy of the REQUEST message that $A$ sends out. $A$ will report FCD relative to only those vehicles that appear in its $r L D M$. Since a single representative node is elected for each VANET radio neighborhood (the maximum 802.11p vehicular radio transmission range $d_{\max }$ being in the order of several hundred meters), the number of LTE channels that are effectively used for the transmission of messages is drastically reduced.

\subsection{Election of representative nodes: multiple connected components case}

Let $T_{\text {cycle }}$ define the duration of the SETUP plus COLLECTION phases. The trigger node starts a new time period by issuing a new REQUEST message every $T_{\text {cycle }}$ seconds. This time period can be broken up into a SETUP phase of duration $T_{\mathrm{S}}$, when representative nodes are elected, and the ensuing COLLECTION phase, when a new set of FCD is sent by current representative nodes every $T_{\mathrm{C}}$ seconds, until the COLLECTION phase is terminated and a new set of representative nodes is to be elected. If the collection phase is repeated $K$ times, then $T_{\text {cycle }}=T_{\mathrm{S}}+K T_{\mathrm{Cs}}$.

Given the maximum number of hops $H$ that the REQUEST message is allowed to traverse (which is related to the ratio between the radius of the target areas and $d_{\text {max }}$; typically $H$ is limited up to few tens), the REQUEST message dissemination delay over the connected component of the VANET that the trigger node belongs to assume a value that lies between $H T_{\min }$ and $H T_{\max }$. Practical values of $T_{\max }$ are in the order of $100 \mathrm{~ms}$. Then, the maximum message dissemination delay is typically below few seconds.

While the trigger node role can be played by suitably scattered RSUs, a simple distributed, OBU based protocol can be defined to trigger the representative node election. The only requisite is that a vehicle that subscribes the service, knows the collection time schedule (i.e., $T_{\mathrm{S}}$ and $T_{\mathrm{C}}$ ) and realizes it is inside the area where the collection service 


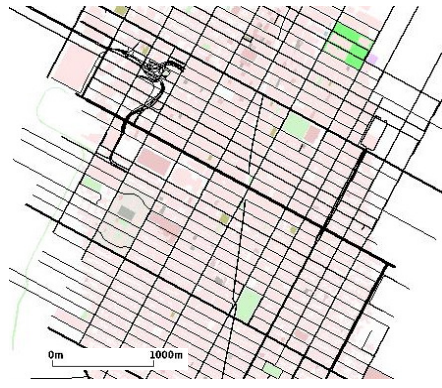

(a) Manhattan District.

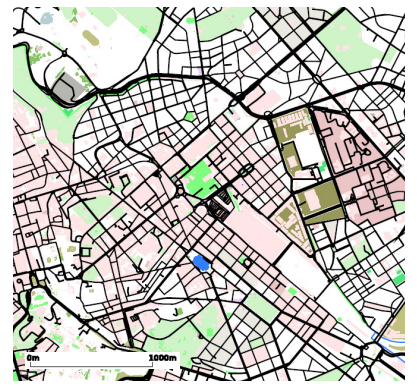

(b) Rome.
Figure 3: Urban scenario maps.

is active. This requisite is easily met by using predefined data stored into the information collection app and the GPS on board the vehicle. At the beginning of each cycle, each vehicle inside the service area sets a timer by choosing a value uniformly at random in the interval $\left[0, T_{\text {trig }}\right]$ and schedules a REQUEST message. If the timer expires and the node has not received any REQUEST message, it sends out its own REQUEST message and elects itself as a representative node. If the node receives the REQUEST message before the trigger timer expires, it cancels its scheduled REQUEST message and schedules the forwarding of the REQUEST message it has just received, by selecting a timer value according to the rule of eq. (1). Then, the protocol proceeds as detailed in the Sec. 3.2 (forwarding and inhibition rules). This fully distributed protocol finds a suboptimal coverage of the vehicle nodes, i.e., the elected representative nodes are in general more than required by a minimum covering set. On the other hand, the mechanism described above is adaptive to the penetration rate of DSRC equipment. It falls back automatically to the case where each single vehicle reports directly its own data via LTE (LTE only approach) as little or no DSRC equipped vehicles are around. On the opposite, as the vehicle density grows, which is the critical case for the cellular network loading, the connectivity of the VANET graph and the distributed procedure outlined above ensure that only a fraction of the vehicle nodes gets elected, as shown in the performance evaluation.

\section{Simulation model}

We evaluate the performance of our proposed mechanisms by using a multi-layer simulation tool that is constructed as a composition of the following simulation modules: SUMO [24], for the vehicular micro-mobility simulation, OMNET ++ [25], for the communication network simulator, and Veins [26], a software module that interconnects SUMO and OMNET++, allowing data import and export between the two.

To define two urban scenarios, we consider actual urban maps of the city centers of Rome and New York, obtained by OpenStreetMap [27]. The first is a part of the district of Manhattan in the city of New York (see Fig. 3(a)). This map is mainly characterized by a regular grid of avenues and streets that create a considerable number of junctions. The second considered scenario covers the neighborhood of Termini Central Station in the city of Rome (Fig. 3(b)). In contrast with the first scenario, this one is characterized by a high level of road layout irregularity and a higher measure of stochastic street orientations. Both considered maps extend over an area of about $12 \mathrm{~km}^{2}$.

Mobility of vehicles is generated by the micro-mobility simulator SUMO, according to the so called "random trips" model. The movement of the vehicles is governed by the car-following model with a target speed of $50 \mathrm{~km} / \mathrm{h}$. According to vehicular traffic features (vehicle density in each road lane, velocity limits, traffic lights) the actual realized velocity can be lower than the target one.

The OMNET ++ tool is used to simulate the behavior of the communication process, including the operations of the Physical, MAC and network layers. The MAC and PHY parameters are set equal to those specified by the IEEE $802.11 p$ standard. We invoke the packet broadcasting operations mode, under which no ACK frames are produced at the MAC layer, as conducted under the IEEE 802.11p MAC specification. We have embedded the implementation of the representative node election logic described in Sec. 3 in the network layer.

As for the VANET, we have jointly used two attenuation models: the Two Exponents Model (TEM) [28] and the Simple Obstacle Shadowing Model (SOSM) [29]. The TEM models the distance dependent component of the power loss: it assumes that the attenuation is $A(d)=\kappa d^{\alpha_{1}}$, for distances $d$ up to a break point value $d_{b p}$. For $d>d_{b p}$, it is $A(d)=\kappa d_{b p}^{\alpha_{1}-\alpha_{2}} d^{\alpha_{2}}$. Typical values of the path loss parameters are $d_{b p}=120 \mathrm{~m}, \alpha_{1}=2$, and $\alpha_{2}=4$. The SOSM reproduces in Veins the shadowing effect of a real urban environment: it describes the attenuation as a function of the depth of the obstacles (e.g., buildings) crossed by radio links. The description of the obstacles in the considered map layouts is taken from the metadata provided by the OSM repository.

We do not have an RSU but we assume that the scenario is based only on OBUs, delegating the role of the trigger node to one or more of these ones as described in Section 3.

Figure 4 shows a snapshot of the vehicle positions in the New York map (circle markers) with the superimposed layout of LTE cellular eNodeBs (triangle markers). We consider two different cases for the placement of LTE eNodeBs: a) eNodeBs located according to a regular hexago-

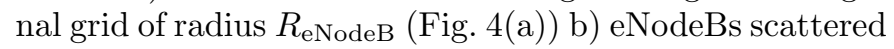
in accordance to a random process, Fig. 4(b).

The COST-Hata model of path loss for urban areas has been used to evaluate the vehicle node Channel Quality Indicator (CQI) and the LTE cell that each vehicle node is associated with (the one with the best detected CQI). 

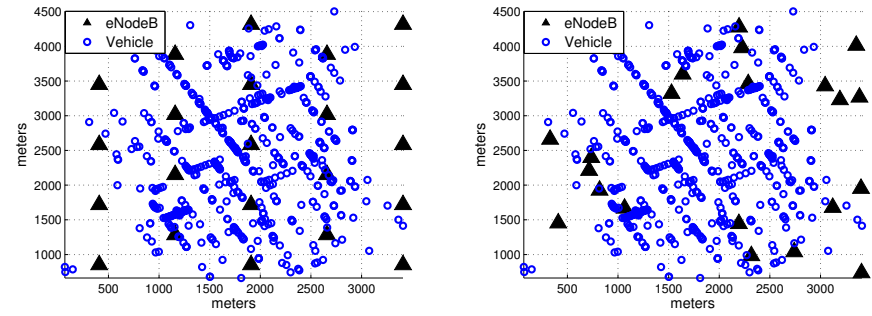

(a) NY, eNodeB placement on an(b) NY, randomly scattered eNhexgonal grid odeBs

Figure 4: Monitored urban area covered by LTE macro-cells; the blue dots represents a snapshot of vehicles in the considered area while black triangles are the ENodeBs (Manhattan, NY).

Table 1: Notations and simulation parameter values

\begin{tabular}{l|l}
\multicolumn{1}{c}{ Parameters } & Values \\
\hline Vehicle density $\lambda\left(\mathrm{veh} / \mathrm{km}^{2}\right)$ & $50-110$ \\
Vehicle target speed $(\mathrm{km} / \mathrm{h})$ & 50 \\
$R_{\text {eNodeB }(\mathrm{m})}$ & $500 \div 3500$ \\
$d_{\max }(\mathrm{LOS})(\mathrm{m})$ & 827 \\
$T_{\min }(\mathrm{ms})$ & 0 \\
$T_{\max }(\mathrm{ms})$ & 100 \\
Hop limit $H$ & 20 \\
Propagation Model for IEEE $802.11 \mathrm{p}$ & $\mathrm{TEM}+\mathrm{SOSM}$ \\
VANET MAC, PHY parameters & IEEE 802.11p \\
IEEE 802.11p Link Rate $(\mathrm{Mbit} / \mathrm{s})$ & 6 \\
IEEE 802.11p tx power $(\mathrm{dBm})$ & 27 \\
Carrier frequency 802.11p $(\mathrm{GHz})$ & 5.9 \\
LTE UE tx power $(\mathrm{dBm})$ & 27 \\
Carrier frequency LTE $(\mathrm{GHz})$ & 0.8
\end{tabular}

The Modulation and Coding Scheme (MCS) is set by each vehicle node transmitting over the LTE channel according to its observed CQI, unless stated otherwise.

As for the LTE, in our model we did not include the data overhead and the association time delay [30] for the connection establishment. This means that for quite large cells (the ones considered in our study) it is assumed that this overhead is negligible since for the time duration of a SETUP and a COLLECTION a vehicle associates only to one ENodeB. We avoid the use of femto and small cells on purpose, to represent the best case in the use of LTE and to measure in this way only the amount of data exchanged for the FCD without the overhead for the cell association. Notice that in case of small and femto cells, due to the vehicle mobility $(13.8 \mathrm{~m} / \mathrm{s})$ a quite large overhead should be instead considered for the LTE association.

Numerical values used for simulation parameters are listed in Tab. 1. Every considered scenario, over a zonal scope of about $12 \mathrm{~km}^{2}$, has been analyzed under different vehicular densities $\lambda$, as reported in Tab. 1.

The baseline solution, taken as a benchmark in the performance comparison, sets a configuration under which each vehicle sends its own CAM directly to the eNodeB using the LTE access network. This solution represents the performance obtained when vehicular data are gathered by using only the LTE network [7][10][11]. Also, it rep- resents the performance behavior of all proposed Hybrid LTE-VANET mechanism during the SETUP phase.

\section{Performance analysis}

\subsection{Performance metrics}

We employ the following performance metrics:

$f_{\mathbf{R V}}$ fraction of all vehicles roaming in the target area that are reached by the REQUEST message propagated in the VANET according to the representative node election logic;

$f_{\mathbf{R N}}$ fraction of all vehicles roaming in the target area that are elected as representative nodes (vehicles that forward the REQUEST message) in the VANET;

$f_{\mathrm{MV}}$ fraction of all vehicles roaming in the target area whose data are reported to the FPS via LTE connections established by the representative nodes;

$D_{\mathbf{R Q}}$ REQUEST message delay: time needed to complete the propagation of the REQUEST message, measured from the instant that this REQUEST message is issued by the trigger node to the time that it has completed its dissemination over the graph component to which the trigger node belongs to;

$M_{\mathbf{C H}}$ number of LTE Physical Uplink Service Channels (PUSCHs) [31] that must be established in a cell to make nodes report their FCD data to the FPS via the LTE network.

$M_{\mathbf{R B}}$ average number of LTE Resource Blocks (RBs) [31] per LTE cell, required by vehicles for communicating over the LTE system;

$L_{\text {data }}$ the average amount of data sent by a representative node over its LTE PUSCH during the SETUP phase.

As for $M_{\mathrm{CH}}$, it is assumed that each node reporting data to the remote server uses a single PUSCH in each COLLECTION instance. Note that a reporting node can aggregate data from other vehicles through the VANET, or it can just report its own data, in case it has no VANET neighbors.

The number $M_{\mathrm{RB}}$ is calculated as follows. All reporting nodes are considered. Let $L_{\mathrm{k}}$ be the amount of data that the $k$-th representative node must report. The spectral efficiency of the $k$-th node is obtained from its CQI level. Let it be $r_{\mathrm{k}}$ bit/RB. Then, the number $m_{\mathrm{k}}$ of RBs required by the $k$-th reporting node is $m_{\mathrm{k}}=\left\lceil L_{\mathrm{k}} / r_{\mathrm{k}}\right\rceil$. Let $N_{\text {eNodeB }}$ be the number of LTE eNodeBs in the scenario and let $\mathcal{R}_{\mathrm{j}}$ denote the set of representative nodes under the coverage of LTE cell $j, j=1, \ldots, N_{\text {eNodeB}}$. Then $M_{\mathrm{RB}}=\frac{1}{N_{\mathrm{eNodeB}}} \sum_{j=1}^{N_{\mathrm{eNodeB}}} \sum_{k \in \mathcal{R}_{\mathrm{j}}} m_{\mathrm{k}}$.

The performance analysis that we carry out accounts for the conduct of the two operations: dissemination of the REQUEST message over the VANET system and vehicle data reporting by the elected representative nodes through the LTE system. 
Table 2: Performance metrics for the dissemination of the REQUEST message in the New York (NY) and Rome (RM) scenarios.

\begin{tabular}{|c|c|c|c|c|c|}
\hline & $\begin{array}{c}\lambda \\
\left(\mathrm{veh} / \mathrm{km}^{2}\right)\end{array}$ & $f_{\mathrm{RV}}$ & $f_{\mathrm{RN}}$ & $f_{\mathrm{MV}}$ & $\begin{array}{c}D_{\mathrm{RQ}} \\
(\mathrm{s})\end{array}$ \\
\hline \multirow{2}{*}{ NY map } & 70 & 0.89 & 0.24 & 0.95 & 0.31 \\
\cline { 2 - 6 } & 110 & 0.90 & 0.19 & 0.98 & 0.40 \\
\hline \multirow{2}{*}{ RM map } & 70 & 0.93 & 0.27 & 0.94 & 0.35 \\
\cline { 2 - 6 } & 87 & 0.93 & 0.22 & 0.94 & 0.48 \\
\hline
\end{tabular}

\subsection{Election of representative nodes by means of the RE- QUEST message}

In the simulation experiments, the trigger node is a randomly selected vehicle. The trigger vehicle is chosen with uniform probability among those roaming in the central part of the considered map. This corresponds to studying the capability of the considered FCD collection protocols in the area surrounding the trigger node.

Performance behavior is assessed by means of evaluation of the metrics $f_{\mathrm{RV}}, f_{\mathrm{RN}}, f_{\mathrm{MV}}$ and $D_{\mathrm{RQ}}$ in the two urban scenarios described in Sec. 4. Results are presented in Tab. 2.

As for the NY map, $f_{\mathrm{RV}}$ is almost insensitive to the vehicle density level and it equals about $90 \%$. The observed values of $f_{\mathrm{RN}}$ range between 0.24 for $\lambda=70 \mathrm{veh} / \mathrm{km}^{2}$ down to 0.19 for $\lambda=110 \mathrm{veh} / \mathrm{km}^{2}$. The fraction of vehicles that serve as representative nodes is thus noted to reduce as the vehicular density $\lambda$ grows, i.e., the efficiency of the aggregation operated by the representative nodes improves with growing levels of $\lambda$.

As for the Rome map, $f_{\mathrm{RV}}$ is again stable with different vehicle density levels. It settles to slightly higher values than with the NY map ( $f_{\mathrm{RV}} \simeq 0.94$ for Rome). Also in this case $f_{\mathrm{RN}}$ decreases with the vehicle density, consistently taking higher values than in the NY case, namely $f_{\mathrm{RN}} \simeq$ 0.27 for $\lambda=70 \mathrm{veh} / \mathrm{km}^{2}, f_{\mathrm{RN}} \simeq 0.22$ for $\lambda=87 \mathrm{veh} / \mathrm{km}^{2}$.

In both NY and Rome cases, the fraction of monitored vehicles $f_{\mathrm{MV}}$ is close to 1 and insensitive to the vehicle density level. In other words, the designated representative nodes do actually represent (cover) essentially all vehicles roaming in the target area.

The dissemination time $D_{\mathrm{RQ}}$ is dependent on the vehicular density level $\lambda$. In the NY scenario, for the lower $\lambda$, it took approximately $310 \mathrm{~ms}$ for the message to reach $89 \%$ of the vehicles. The message dissemination delay increases to $400 \mathrm{~ms}$ for the higher $\lambda$ level. The corresponding values for the Rome scenario are between 350-480 ms.

The higher levels of delay and $f_{\mathrm{RN}}$ observed in the Rome map are due to the irregularity of the street layout that is noted to have lower vehicular communications connectivity, so that a larger number of hops are needed to reach out vehicles distant from the trigger node point.

\subsection{Load on LTE cellular system during the SETUP phase}

Once the representative nodes are elected, they proceed to report the FCD of the vehicles roaming in the region of interest. We investigate the case where the reported FCD
Table 3: $L_{\text {data }}$ for two different vehicular density levels and for four different LTE eNodeB distance $R_{\text {eNodeB }}$ in the New York (NY) and Rome (RM) scenarios.

\begin{tabular}{|c|c|c|c|c|c|}
\hline & & \multicolumn{4}{|c|}{$\begin{array}{c}L_{\text {data }} \\
{[\mathrm{kB}]}\end{array}$} \\
\hline \multirow{2}{*}{ Map } & $\begin{array}{c}\lambda \\
{\left[\mathrm{veh} / \mathrm{km}^{2}\right]}\end{array}$ & $\begin{array}{c}R_{\text {eNodeB }} \\
500 \mathrm{~m}\end{array}$ & $\begin{array}{c}R_{\text {eNodeB }} \\
1500 \mathrm{~m}\end{array}$ & $\begin{array}{c}R_{\text {eNodeB }} \\
2500 \mathrm{~m}\end{array}$ & $\begin{array}{c}R_{\text {eNodeB }} \\
3500 \mathrm{~m}\end{array}$ \\
\hline \multirow{2}{*}{$\mathrm{NY}$} & 70 & 1.795 & 12.053 & 23.988 & 24.090 \\
\cline { 2 - 6 } & 110 & 3.733 & 20.970 & 41.671 & 41.926 \\
\hline Rome & 70 & 1.721 & 9.267 & 20.653 & 24.218 \\
\cline { 2 - 6 } & 87 & 1.490 & 7.591 & 16.331 & 19.162 \\
\hline
\end{tabular}

data contains the vehicles' geographical positions. Under our approach, each representative node sends a REPORT message with its own FCD and the positions of the vehicles whose IDs are listed in the $r L D M$ built during the REQUEST dissemination phase (see Sec. 3.2). The REPORT message sent by each representative node consists of:

- network plus transport headers (IPv6+UDP) of 48 B (see Table 1 in [7]);

- an application level header of $48 \mathrm{~B}$, that contains the representative node ID, its position and the same data as envisaged in the Vehicle High Frequency Container of the $\mathrm{CAMs}^{2}$; moreover, it contains also the number $n \geq 0$ of ensuing records, relevant to neighbor vehicles' data;

- a list of records: each record has a length of $32 \mathrm{~B}$, and it is made up of: i) a $1 \mathrm{~B}$ sequence number; ii) a $17 \mathrm{~B}$ encoding of the 17 characters US NHTSA standard Vehicle Identification Number; iii) the position of the reported vehicle, encoded with $14 \mathrm{~B}$.

Overall, a REPORT message containing data from $n$ neighborhood vehicles has a length of $96+32 n \mathrm{~B}$. The size values of $L_{\text {data }}$ for New York and Rome are presented in Tab. 3. It is apparent that the average amount of data that each representative node has to transfer over the LTE connection grows quickly with the area covered by an LTE eNodeB.

We investigate the performance behavior of the urban scenarios by varying the value of the LTE eNodeB distance $R_{\text {eNodeB }}$ and by considering different vehicular densities. The crucial points are: i) the overhead implied by setting up and maintaining an active LTE connection, hence the number of used LTE channels per cell; ii) the load seen by an LTE eNodeB due to the overall number of vehicle nodes under its coverage that require an LTE channel.

The impact of the vehicle data transfer through the LTE access network is highlighted by the results in Figs. 5

\footnotetext{
${ }^{2}$ Our setting is consistent with [7], where it is mentioned that the maximum length of a CAM containing only the mandatory fields, including the Basic Container and the Vehicle HF Container, is 50 bytes.
} 

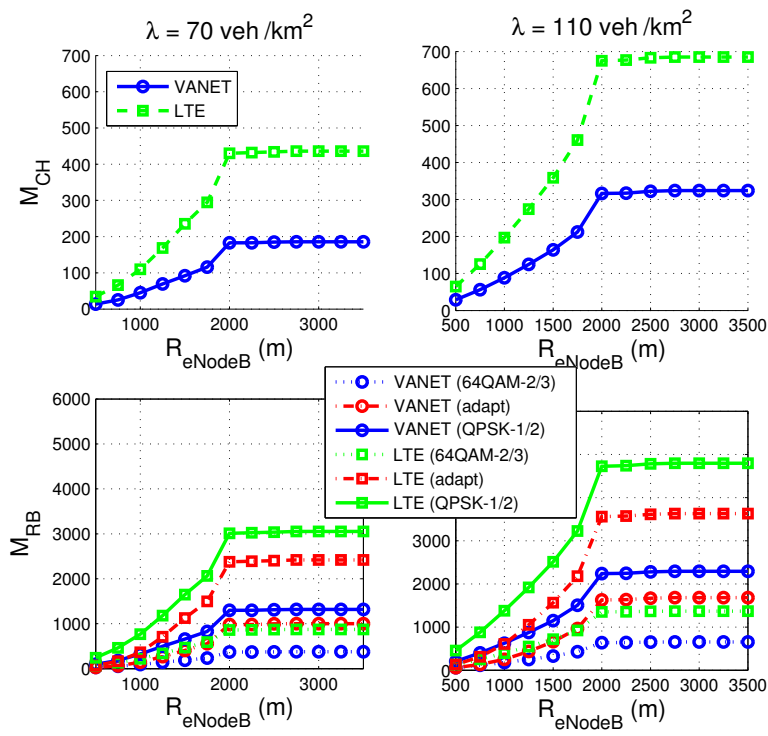

O.. VANET (64QAM-2/3)

VANET (QPSK-1

LTE (64QAM-2/3)

LTE (adapt) LTE (QPSK-1/2)

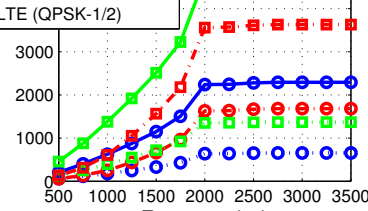

$\mathrm{R}_{\text {eNodeB }}(\mathrm{m})$

Figure 5: Average number of LTE uplink radio channels used per cell, $M_{\mathrm{CH}}$, (top graphs) and average number of uplink RBs used per cell, $M_{\mathrm{RB}}$, (bottom graphs) vs. the eNodeB transmission range $R_{\text {eNodeB for two different vehicular density levels and for the New }}$ York scenario.

and 6. The metrics $M_{\mathrm{CH}}$ and $M_{\mathrm{RB}}$ are plotted as a function of the inter-eNodeB distance, $R_{\text {eNodeB }}$, for the NY map (Fig. 5) and the Rome map (Fig. 6). In these figures, we compare two approaches: i) each vehicle sends its own data individually, by using its own dedicated LTE connection (curves labelled with LTE); ii) our proposed protocol is used, representative nodes are elected and only those nodes report data about themselves and about their respective neighbors via their LTE connections, as described in Sec. 3.2 (curves labelled with VANET).

As for $M_{\mathrm{CH}}$, under the LTE approach (curves with the square marker), the number of LTE channels assigned by an eNodeB to report FCD to the FPS is equal to the total number of vehicles under the coverage area of the eNodeB. This grows quickly as the area covered by a single eNodeB expands. In comparison with the $L T E$ approach, we note that the VANET scheme (curves with circle markers) is able to reduce the number of nodes elected to report vehicles' data via the LTE access network, leading to a substantial reduction of number of required LTE channels per cell. The presented curves flatten for growing values of $R_{\text {eNodeB }}$, since eventually only a single LTE eNodeB covers most of the considered map area. When a single cell covers most of vehicles, further increments of $R_{\text {eNodeB }}$ do not change the load of the single LTE cell in the scenario.

To expand the performance evaluation as the vehicle density and the eNBs layout are varied, in the case of the New York map we have set up specific models. eNBs positions have been generated according to a hard-core spatial random process, namely Matern type II [32]. It is
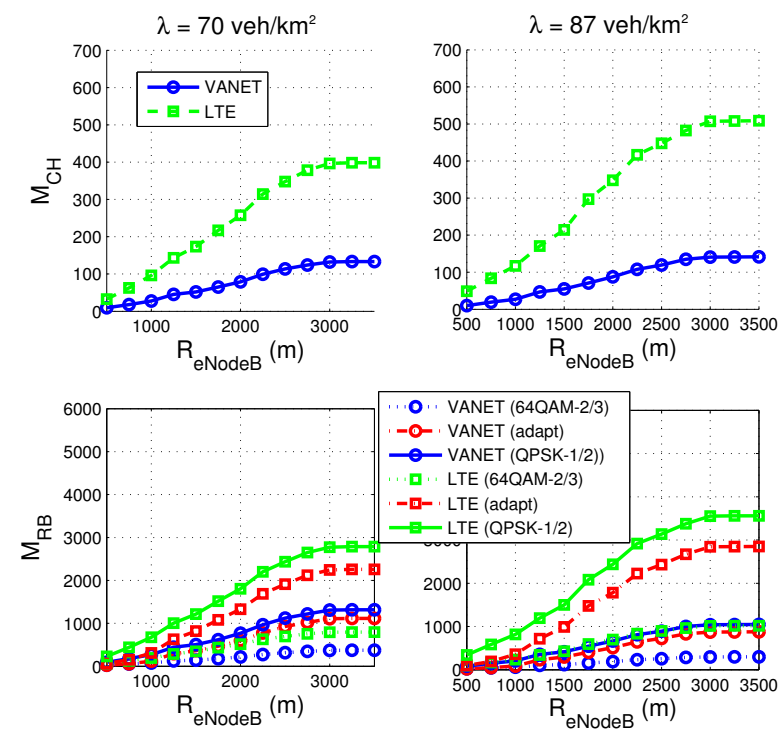

Figure 6: Average number of LTE uplink radio channels used per cell, $M_{\mathrm{CH}}$, (top graphs) and average number of uplink RBs used per cell, $M_{\mathrm{RB}}$, (bottom graphs) vs. the eNodeB transmission range $R_{\text {eNodeB }}$ for two different vehicular density levels and for the Rome scenario.

constructed starting from a uniform spatial Poisson Point Process with mean density $\mu_{b}$. Then points are assigned with random marks drawn form a uniform probability distribution over $[0,1]$. Points having a neighbor within distance $d$ with a mark level less then their own are labelled with a '0'. After removing all points labelled with a '0', the residual points cannot be closer than the chosen distance $d$. The relevant mean density $\mu$ is

$$
\mu=\frac{1-e^{-\mu_{b} \pi d^{2}}}{\pi d^{2}}
$$

If the eNBs were laid out according to a regular hexagonal grid of radius $R_{\text {eNodeB }}$, the resulting density would be $\mu=\frac{2}{3 \sqrt{3} R_{\mathrm{eNodeB}}^{2}}$. This value can be plugged into eq. (2), hence the value of $\mu_{b}$ can be found, given $d$. In our simulations we set $d=100 \mathrm{~m}$ and let $R_{\mathrm{eNodeB}}$ vary from $200 \mathrm{up}$ to $3000 \mathrm{~m}$. Vehicle positions are obtained from the SUMO simulation of the New York area, as for Fig. 4. To let the vehicle nodes vary, we sample vehicles with probability $p$, i.e., we assume that only a fraction $p$ of the vehicles moving in the considered area take part in the traffic information collection. Hence, the vehicle node density is $p \lambda$, where $\lambda$ is the average density of all vehicles. Vehicle nodes are associated to the closest eNB, i.e., the serving eNB is chosen as the one having the least average path loss to the vehicle node.

In these simulations we have used the fully distributed trigger node procedure outlined in Sec. 3.3, since we vary the vehicle density and hence we consider cases where the VANET graph is sparse and disconnected. 


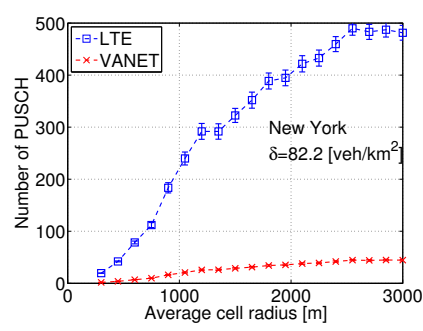

(a)

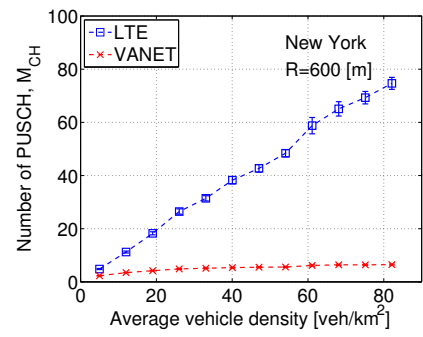

(b)
Figure 7: Average number of LTE uplink radio channels used per cell, $M_{\mathrm{CH}}$ under randomised eNodeB positions: (a) varying average cell radius; (b) varying vehicle node density.

Figure 7 (a) plots $M_{\mathrm{CH}}$ as a function of the average cell radius for the case of randomly scattered eNBs for an average density of vehicle of $82.2 \mathrm{veh} / \mathrm{km}^{2}$. Figure $7(\mathrm{~b})$ plots $M_{\mathrm{CH}}$ as a function of the average vehicle node density, obtained by vehicle sampling as explained above; the average cell radius is $600 \mathrm{~m}$. Blue square markers refer to the case where only the LTE cellular network is used for the vehicle data collection, while the red cross markers correspond to our protocol, based on VANET level election of representative nodes that are the only one using an LTE channel. $95 \%$ level confidence intervals are shown as well.

The LTE only approach faces a significant growth of the number of required LTE channels as both the average cell radius or the vehicle node density are increased. In both cases, the average number of vehicle nodes served by a same eNodeB grows. On the other hand, electing representative nodes is quite effective in reducing the number of required channels. As the vehicle node density gets smaller, the VANET gets more disconnected and the VANET level aggregation is less effective: this is highlighted by the convergence of the values of $M_{\mathrm{CH}}$ obtained with LTE and VANET for low vehicle density levels in Fig. 7(b). From the point of view of the load on the cellular network, the low density case is the least critical. Our proposed approach based on the VANET falls back to the basic LTE only solution as the vehicle node density lowers. This occurs seamlessly, without having the vehicle estimate the mean density or any other global parameter. In other words, our proposed approach is robust with respect to the vehicle node density, yielding an occupation of LTE channels that is never bigger than the LTE only approach, and gets much smaller as the vehicle node density grows.

The major offload brought about by the use of the VANET is highlighted by the performance curves shown in Figs. 5 and 6 for $M_{\mathrm{RB}}$, the average number of RBs used by representative nodes in each LTE cell, as a function of $R_{\text {eNodeB }}$. We identify two performance bounds: i) the best case, when each node using an LTE channel is able to use the high rate MCS, namely $64 \mathrm{QAM}$ with code rate $2 / 3$; ii) the worst case, when every node using an LTE channel must use of lowest rate MCS, namely QPSK with
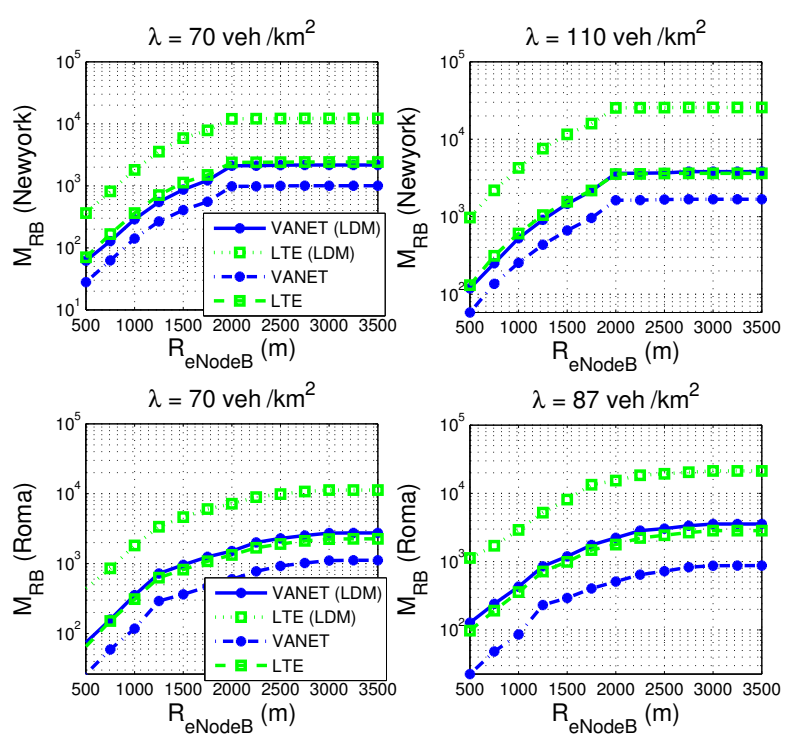

Figure 8: Average number of RBs per cell, $M_{\mathrm{RB}}$, used by nodes transmitting over the LTE channels vs. $R_{\mathrm{eNodeB}}$ for two density levels, NY map (top graphs) and Rome map (bottom graphs). The green curves (square markers) refer to the case where full connectivity information is transferred, in addition to node positions. The blue curves (circle markers) correspond to the case where only nodal position information is transferred.

code rate $1 / 2$. Besides those bounds, we also evaluate the intermediate case, where each node using an LTE channel measures its CQI and infers what is the best MCS that it can use (curves labelled with adapt). The worst performance exhibited under the VANET approach is close to the best performance obtained under the LTE scheme for the highest $\lambda$. The performance gap between the corresponding bounds and between the two approaches (e.g., as measured by the adaptive case) broadens as the number of eNodeBs is reduced. This is a critical issue, since low intensity data collection of FCD should be taken care of by macro-cells, rather than by hot spot micro-cells, intended to boost the capacity offered in special areas for broadband users. On the other hand, macro-cells cover urban areas that can encompass hundreds of vehicles. Hence, the VANET scheme proposed herein is highly effective in supporting massive FCD collection.

Another performance advantage offered by the proposed approach is appreciated by examining the results shown in Fig. 8. The metric $M_{\mathrm{RB}}$ is plotted vs. $R_{\mathrm{eNodeB}}$ for two different vehicular density levels, in the NY and Rome scenarios. We compare the adaptive LTE channel performance obtained under the VANET and LTE approaches under two alternative cases: i) only vehicular positions are reported to the FPS via LTE (the same case as the one shown in Figs. 5 and 6); ii) both vehicular positions and VANET connectivity information are reported 
to the FPS (curves denoted with VANET (LDM) and LTE $(L D M))$. The latter case is appealing for a centralized optimization of inter-vehicular communications, e.g., for content distribution; in general, whenever the knowledge of the VANET topology can be exploited profitably.

It is noted that the advantage of our approach is enhanced when it is required to transfer information that includes nodal positions as well as their connectivity relationships within the VANET. In fact, under our VANET approach, this amounts to transfer the full list of neighboring nodal IDs and positions, rather than only those listed in the reduced table $r L D M$. Hence, the difference is impacted by the number of common neighbors of adjacent representative nodes. Under the LTE framework, the knowledge of the VANET connectivity requires each vehicular node to report information about itself plus the full list of its neighbor's IDs and positions.

\subsection{Load on LTE cellular system during the COLLEC- TION phase}

In this section, we study the impact of the vehicular data gathering on the LTE access network during the COLLECTION phase. We compare three approaches (the acronyms are used as labels in the graphs):

$\boldsymbol{L T E}$ Each vehicle sends its own FCD information directly to eNodeB in a dedicated LTE channel.

$\boldsymbol{V A N E T}$ Representative nodes are elected by means of a VANET driven process (the REQUEST message dissemination described in Sec. 3.1). Then, representative nodes are responsible to send their $r L D M$ to their covering eNodeBs, thus reporting their own FCD plus those of part of their vehicular neighborhood.

$\boldsymbol{H Y B R I D}$ This is a state-of-the-art Hybrid approach as reviewed in Sec. 2.2. The information about all monitored vehicles in the target area, collected at the FPS during the SETUP phase, is used to synthesize a set of cluster-head nodes that cover all the target area. The designated cluster-heads are responsible to aggregate and send the FCD of their respective vehicular neighborhoods. Note that the identification of a set of cluster-head nodes covering all other vehicles requires the FPS to acquire the entire VANET connective graph.

More in depth, we have implemented the following scheme to select cluster-heads according to the HYBRID approach. First we order the vehicle nodes inside the coverage area of a eNodeB by decreasing levels of CQI. The node with the highest CQI level is elected cluster-head. This node and all its neighbors are removed from the list of the nodes under the coverage of the considered eNodeB. We iterate this selection process until the list is empty. Note that the cluster-heads are elected on the basis on their LTE channel quality, maximizing the cluster-head LTE radio
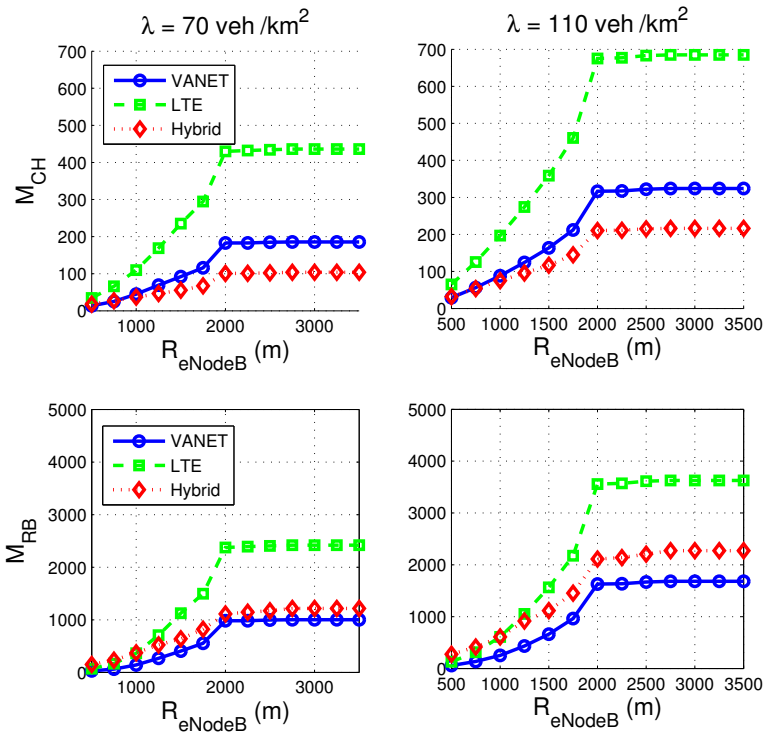

Figure 9: Average number of LTE uplink radio channels used per cell, $M_{\mathrm{CH}}$, (top graphs) and average number of uplink RBs used per cell, $M_{\mathrm{RB}}$, (bottom graphs) vs. the eNodeB transmission range $R_{\text {eNodeB }}$ for two different vehicular density levels and for the New York scenario.

capacity. Moreover, the election process guarantees that there is no duplication of FCD reported to the FPS, thus minimizing the overall amount of information to be sent through LTE channels. Clearly, the implementation of the cluster-head election according to the Hybrid process requires that full VANET topology information be collected during the SETUP phase. MCS is set according to the transmitting node CQI.

Figures 9 and 10 compare the load induced on the LTE access network by the three data collection approaches listed above: LTE (green square markers); VANET (blue circle markers); HYBRID (red diamond markers).

The obvious result shown in Figs. 9 and 10 is that using VANET communications (as done in the VANET or $H Y$ $B R I D$ approaches) we can drastically reduce the number of PUSCHs and RBs occupied in each LTE cell, in comparison with the LTE approach. The performance gap between the LTE and the other two approaches broadens as the number of eNodeBs is reduced. These results make a strong case for the exploitation of peer-to-peer communication networks among vehicle nodes, as allowed by the DRSC VANET, to aggregate FCD before sending them through the LTE cellular network.

The less obvious result is that HYBRID turns out to use a smaller number of LTE channels with respect to $V A N E T$, whereas the latter consumes a smaller amount of RBs to carry the FCD with respect to $H Y B R I D$. This apparent contradiction is explained as follows. According to $H Y B R I D$ algorithm, representative nodes are chosen so as to obtain a sparse, yet full coverage of the vehicle 

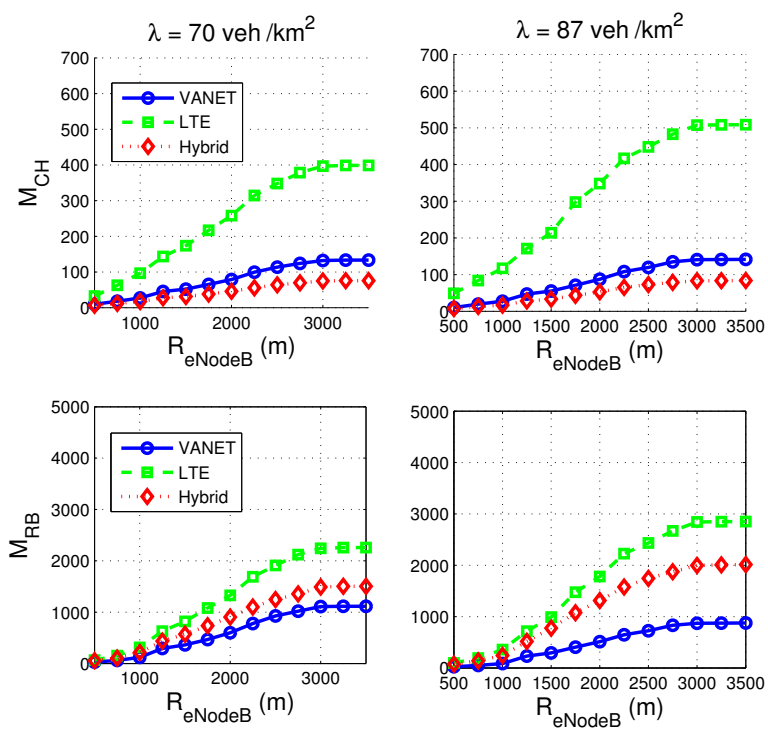

Figure 10: Average number of LTE uplink radio channels used per cell, $M_{\mathrm{CH}}$, (top graphs) and average number of uplink RBs used per cell, $M_{\mathrm{RB}}$, (bottom graphs) vs. the eNodeB transmission range $R_{\text {eNodeB }}$ for two different vehicular density levels and for the Rome scenario.

nodes in the target area. There is no requirement that representative nodes form a connected network, only that each given vehicle node can communicate with one representative node. On the other hand, with VANET the identification of the representative nodes is driven by the dissemination logic, hence they form a connected set. As a matter of example, in a span of road of length $L$, the number of representative nodes is in the order of $L /\left(2 d_{\max }\right)$ with $H Y B R I D$ while it is in the order of $L / d_{\max }$ in case of VANET. Conversely, the number of vehicle nodes that each representative node has to report on is smaller with VANET than with HYBRID, hence less RBs are enough to carry the FCD in case of VANET with respect to $H Y$ BRID.

\subsection{Load on LTE cellular system with multiple OBU orig- inators}

In this section, we study the impact of our VANET solution when more than one vehicle generate the REQUEST message. This is the case with the election protocol outlined in Sec. 3.3. Note that REQUEST messages are instrumental to identify the elected representative nodes, hence the originating trigger node is irrelevant. This implies that a REQUEST message originated from source node $X$ can inhibit a vehicle node that has received a $\mathrm{RE}$ QUEST message originated by another node $Y$ and is currently running its timer.

We consider the same simulation scenarios as in Sec. 5.4 for New York and Rome and for two different vehicular density levels. In each scenario we select randomly $n_{\mathrm{t}}$
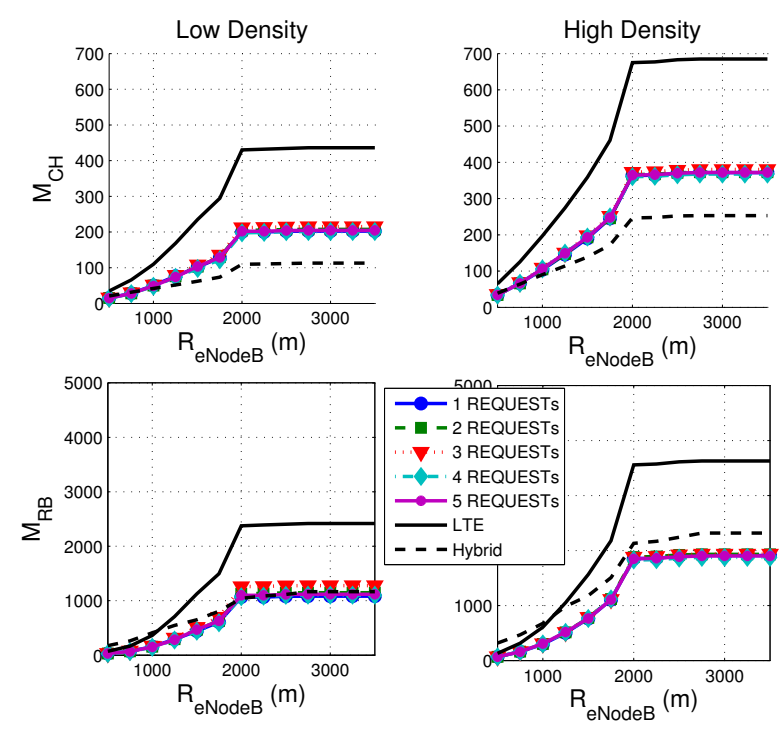

Figure 11: Average number of LTE uplink radio channels used per cell, $M_{C H}$, (top graphs) and average number of uplink RBs used per cell, $M_{R B}$, (bottom graphs) vs. the eNodeB transmission range $R_{\text {eNodeB }}$ for two different vehicular density levels during the COLLECTION phase in New York.

trigger vehicle nodes that are responsible to generate a REQUEST message. The trigger vehicle nodes are chosen randomly in different areas of the map, e.g., in case of 4 trigger nodes the map is divided into four quarters and one vehicle node is elected randomly within each map quarter. We have run simulations for $n_{\mathrm{t}}=1, \ldots, 5$.

Figure 11 shows the performance of our proposed approach (VANET), compared with the LTE and HYBRID solutions, during the COLLECTION phase in New York. The plotted metrics are the number of LTE channels and RBs used by nodes sending FCD via the LTE access network, according to the three approaches. The Fig. 12 show the same performance metrics in case of the Rome map. The number of trigger vehicle nodes originating REQUEST messages is annotated in the graph and ranges between 1 and 5 .

The approaches that make use of the VANET communication links, namely VANET and HYBRID, attain performance levels close to one another. They definitely outperform the LTE solution. Also in this case, we can observe a trade-off between VANET and HYBRID in terms of $M_{\mathrm{CH}}$ and $M_{\mathrm{RB}}$, as in the case of a single trigger node.

The main result that we can deduce from this set of simulations is the low sensitivity of the considered performance metrics with respect to the number of the REQUEST originators. This is particularly important for the following considerations. In our approach, we use the VANET to disseminate the so called REQUEST message to trigger the FCD collection process and, as we have shown in our previous work [6], this mechanism works well 

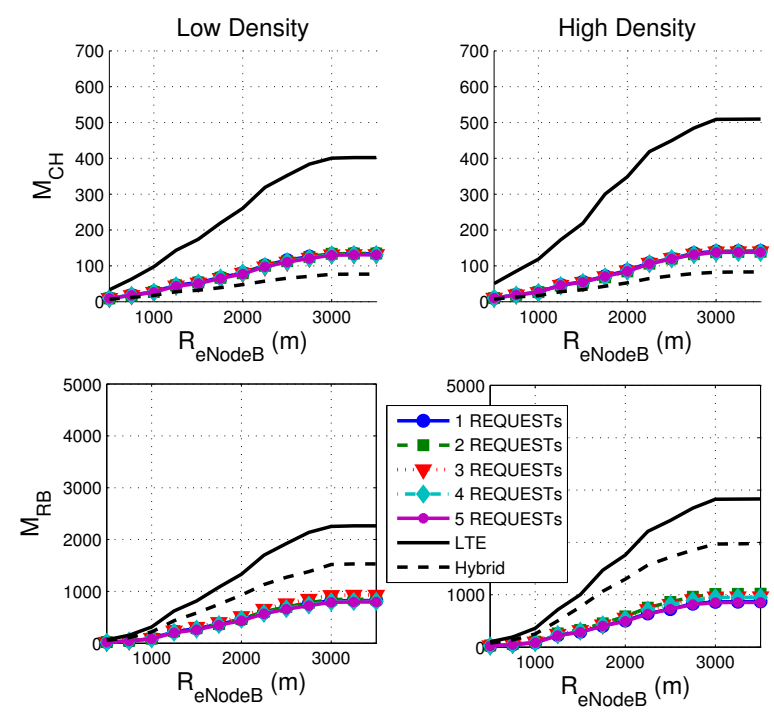

Figure 12: Average number of LTE uplink radio channels used per cell, $M_{C H}$, (top graphs) and average number of uplink RBs used per cell, $M_{R B}$, (bottom graphs) vs. the eNodeB transmission range $R_{\text {eNodeB }}$ for two different vehicular density levels during the COLLECTION phase in Rome.

when the REQUEST generation is centrally performed using a fixed access point called Road Side Unit (RSU). In this paper, we have considered the case when no specific additional VANET infrastructure node is present, delegating the whole work to the moving vehicles. The first set of simulation shows that our idea is also able to outperform traditional solutions, like LTE and HYBRID, to off-load the cellular network. Now, we show that the centralized scenario vision guaranteed by a RSU is not necessary for a good REQUEST dissemination process, or in other words, vehicles can be directly used to trigger the FCD collection process. Also, we are not bounded to use a particular stringent algorithm with a full knowledge of the network, being able to select the "best" vehicle node among a multitude. On the contrary, the impact of having multiple trigger nodes scattered at random in the target area is marginal, i.e., the grid of representative nodes that emerges out of the REQUEST message dissemination exhibits robust performance levels with respect to the position of the initial trigger nodes.

\section{Conclusions}

We have presented an FCD collection protocol via LTE cellular network, where substantial offloading is obtained by resorting to $\mathrm{V} 2 \mathrm{~V}$ direct communication links to elect representative vehicle nodes that aggregate FCD of their respective neighboring vehicle nodes before sending them through LTE channels. The identification of representative nodes is distributed, based on autonomous rules fol- lowed by each participating vehicle node, seamless to the LTE cellular network. V2V communication is assumed to take place by means of the IEEE 802.11p VANET, since this is the technology specifically designed for that purpose and that kind of transponders are expected to become part of the vehicle equipment, at least because of safety requirements. The proposed solution adapts fully to the available penetration rate of the VANET equipment; it falls back automatically to LTE only FCD collection in case VANET equipment is not available or excessively sparse.

Further lines of research are: (i) the possibility of exploiting alternative technologies to provide V2V communication to carry out the task of representative vehicle node election, e.g., LTE D2D communications, WiFi, VLC, mmWave; (ii) the achievable throughput of the FCD collection in view of the enormous amount of data that could be profitably obtained by roaming vehicles, both as regards their status (position, kinematic parameters, state of maintenance, administrative data, usage of vehicle functions) and the surrounding environment (state of road pavement, air quality, climatic data, images from vehicle videocameras, vehicular traffic congestion, vehicular traffic OD patterns, parking information, public transportation schedule, freight logistics). The achievable throughput of FCD collection is a key issue given the enormous amount of sensor data that can be potentially drawn from roaming vehicles, e.g., up to $100 \mathrm{Mbit} / \mathrm{s}$ can be transferred on the CAN bus of a vehicle [33]. This extreme real time, high resolution big data calls for new ideas on distributed processing, and networking to pave the way to smart applications, a trend that is still in its infancy.

\section{References}

[1] T. Darwish, K. A. Bakar, Traffic density estimation in vehicular ad hoc networks: A review, Ad Hoc Networks 24, Part A (2015) $337-351$.

[2] S. Djahel, R. Doolan, G.-M. Muntean, J. Murphy, A Communications-Oriented Perspective on Traffic Management Systems for Smart Cities: Challenges and Innovative Approaches, IEEE Communications Surveys Tutorials 17 (1) (2015) 125-151.

[3] European Telecommunications Standards Institute ETSI TS 302 637-2 v1.3.0; Intelligent Transport Systems (ITS); Vehicular Communications; Basic Set of Applications; Part 2: Specification of Cooperative Awareness Basic Service (August 2013).

[4] IEEE, IEEE Guide for Wireless Access in Vehicular Environments (WAVE) - Architecture, Std 1609.0-2013, IEEE (Mar. 2014).

[5] F. Dressler, H. Hartenstein, O. Altintas, O. Tonguz, Intervehicle communication: Quo vadis, IEEE Communications Magazine 52 (6) (2014) 170-177.

[6] P. Salvo, I. Turcanu, F. Cuomo, A. Baiocchi, I. Rubin, LTE floating car data application off-loading via VANET driven clustering formation, in: 12th Annual Conference on Wireless Ondemand Network Systems and Services (WONS), 2016, pp. 1-8.

[7] J. Calabuig, J. Monserrat, D. Gozalvez, O. Klemp, Safety on the Roads: LTE Alternatives for Sending ITS Messages, IEEE Vehicular Technology Magazine 9 (4) (2014) 61-70.

[8] S. Uppoor, M. Fiore, Characterizing Pervasive Vehicular Access to the Cellular RAN Infrastructure: An Urban Case Study, IEEE Trans. on Vehicular Technology 64 (6) (2015) 2603-2614. 
[9] S. Ancona, R. Stanica, M. Fiore, Performance boundaries of massive Floating Car Data offloading, in: 11th Annual Conference on Wireless On-demand Network Systems and Services (WONS), 2014, pp. 89-96.

[10] G. Araniti, C. Campolo, M. Condoluci, A. Iera, A. Molinaro, LTE for vehicular networking: a survey, IEEE Communications Magazine 51 (5) (2013) 148-157.

[11] Z. Hameed Mir, F. Filali, LTE and IEEE 802.11p for vehicular networking: a performance evaluation, EURASIP Journal on Wireless Communications and Networking 2014 (1) (2014) 115.

[12] M. Gramaglia, M. Calderon, C. Bernardos, ABEONA Monitored Traffic: VANET-Assisted Cooperative Traffic Congestion Forecasting, IEEE Vehicular Technology Mag. 9 (2) (2014) 5057.

[13] Y. Dieudonne, B. Ducourthial, S. M. Senouci, COL: A data collection protocol for VANET, in: IEEE Intelligent Vehicles Symposium (IV), 2012, pp. 711-716. doi:10.1109/IVS.2012. 6232266.

[14] G. Remy, S.-M. Senouci, F. Jan, Y. Gourhant, LTE4V2X: LTE for a Centralized VANET Organization, in: IEEE Global Telecommunications Conference (GLOBECOM 2011), 2011, pp. $1-6$.

[15] S. Jia, S. Hao, X. Gu, L. Zhang, Analyzing and relieving the impact of FCD traffic in LTE-VANET heterogeneous network, in: 21st International Conference on Telecommunications (ICT), 2014, pp. 88-92.

[16] R. Sivaraj, A. Gopalakrishna, M. Chandra, P. Balamuralidhar, QoS-enabled group communication in integrated VANETLTE heterogeneous wireless networks, in: 7th IEEE International Conference on Wireless and Mobile Computing, Networking and Communications (WiMob), 2011, pp. 17-24. doi : 10.1109/WiMOB. 2011.6085417.

[17] P. M. d'Orey, N. Maslekar, I. de la Iglesia, N. K. Zahariev, NAVI: Neighbor-Aware Virtual Infrastructure for Information Collection and Dissemination in Vehicular Networks, in: 81st IEEE Vehicular Technology Conference (VTC Spring), 2015, pp. 1-6.

[18] M. De Felice, F. Cuomo, A. Baiocchi, I. Turcanu, S. Zennaro, Traffic Monitoring and Incident Detection Using Cellular and Early Stage VANET Technology Deployment, in: Proceedings of the First International Workshop on Internet of Vehicles and Vehicles of Internet, IoV-VoI '16, ACM, Paderborn, Germany, 2016, pp. 1-6. doi:10.1145/2938681.2938685.

[19] European Telecommunications Standards Institute ETSI TS 302 636-4-1 v1.2.1; Intelligent Transport Systems (ITS); Vehicular Communications; GeoNetworking; Part 4, Geographical addressing and forwarding for point-to-point and pointto-multipoint communications; Sub-part 1: Media-Independent Functionality (July 2014).

[20] H. T. Cheng, H. Shan, W. Zhuang, Infotainment and road safety service support in vehicular networking: From a communication perspective, Mechanical Sys. and Signal Proc. 25 (6).

[21] C. Sommer, F. Dressler, Vehicular Networking, Cambridge University Press, 2014. doi:10.2277/1107046718.

[22] M. Chaqfeh, A. Lakas, I. Jawhar, A survey on data dissemination in vehicular ad hoc networks, Vehicular Communications 1 (4) (2014) $214-225$.

[23] European Telecommunications Standards Institute ETSI EN 302 636-3 v1.2.1; Intelligent Transport Systems (ITS); Vehicular Communications; GeoNetworking; Part 3, Network architecture (December 2014).

[24] D. Krajzewicz, C. Rossel, Simulation of Urban MObility (SUMO), German Aerospace Centre, available at: http://sumo.sourceforge.net/index.shtml (2002).

[25] A. Varga, R. Hornig, An overview of the OMNeT++ simulation environment, in: 1st ACM/ICST International Conference on Simulation Tools and Techniques for Communications, Networking and Systems (SIMUTools 2008), ACM, Marseille, France, 2008.

[26] C. Sommer, R. German, F. Dressler, Bidirectionally Coupled
Network and Road Traffic Simulation for Improved IVC Analysis, IEEE Transactions on Mobile Computing 10 (1) (2011) $3-15$.

[27] OpenStreetMap, available at: http://www.openstreetmap.org/ (2001).

[28] K. L. H. Hartenstein, VANET Vehicular Applications and InterNetworking Technologies (Intelligent Transport Systems), John Wiley \& Sons, 2010.

[29] C. Sommer, D. Eckhoff, R. German, F. Dressler, A computationally inexpensive empirical model of IEEE $802.11 \mathrm{p}$ radio shadowing in urban environments, in: 8th International Conference on Wireless On-Demand Network Systems and Services (WONS), 2011, pp. 84-90.

[30] G. C. Madueo, J. J. Nielsen, D. M. Kim, N. K. Pratas, . Stefanovi, P. Popovski, Assessment of lte wireless access for monitoring of energy distribution in the smart grid, IEEE Journal on Selected Areas in Communications 34 (3) (2016) 675-688. doi: 10.1109/JSAC. 2016.2525639.

[31] European Telecommunications Standards Institute ETSI TS 136213 V12.4.0; LTE; Evolved Universal Terrestrial Radio Access (E-UTRA); Physical layer procedures (3GPP TS 36.213 version 12.4.0 Release 12) (February 2015).

[32] M. Haenggi, Stochastic Geometry for Wireless Networks, Cambridge University Press, 2012.

[33] S. Kang, S. Han, S. Cho, D. Jang, H. Choi, J. W. Choi, High speed CAN transmission scheme supporting data rate of over $100 \mathrm{Mb} / \mathrm{s}$, IEEE Communications Magazine 54 (6) (2016) 128135. 